EPISTEME KOINONIA

Revista Electrónica de Ciencias de la Educación, Humanidades, Artes y Bellas Artes

Año I. Vol I. N².

Julio - Diciembre, 2018

Hecho el depósito de Ley: FA2018000022

ISSN: 2665-0282

FUNDACIÓN KOINONIA (F.K).

Santa Ana de Coro, Venezuela

Raemy Roque Díaz

\title{
Desarrollo personal y manejo asertivo de emociones en estudiantes
}

\section{Personal development and assertive management of emotions in students}

\author{
Raemy Roque Díaz \\ raemyrdiaz@gmail.com \\ Centro Experimental de Atención Integral al Niño, Santa Ana de Coro \\ Venezuela
}

Recibido: 15 de abril de 2018

Aprobado: 1 de junio de 2018

\section{RESUMEN}

Se procuró contribuir en la transformación de un grupo de estudiantes de sexto grado en relación a lo planteado, debido que presentan inconvenientes para relacionarse asertivamente con sus compañeros, originándose así en el ambiente de aprendizaje comportamientos que no le permiten explotar al máximo sus capacidades. Se aborda desde la teoría crítica e investigación acción participativa para promover la transformación de los participantes, mediante la aplicación de encuentros donde se procura el crecimiento personal, así como fomentar la creatividad y el pensamiento crítico, siendo esta una oportunidad para crecer personal y profesionalmente desde la praxis pedagógica de la orientación educativa. Se evidencia una evolución cognitiva conductual por parte de los estudiantes de sus emociones, en comparación con el momento inicial de la investigación, sin embargo, es pertinente seguir trabajando con ellos en aras de contribuir al logro de una personalidad asertivamente emocional.

Descriptores: psicología de la educación, psicología del niño, orientación pedagógica, asesoramiento.

\begin{abstract}
An effort was made to contribute to the transformation of a group of sixth-grade students in relation to the above, since they present inconveniences to relate assertively with their peers, thus originating behaviors in the learning environment that do not allow them to fully exploit their abilities. It is approached from the critical theory and participatory action research to promote the transformation of the participants, through the application of meetings where personal growth is sought, as well as fostering creativity and critical thinking, this being an opportunity to grow personally and professionally from the pedagogical praxis of the educational orientation. A cognitive - behavioral evolution is
\end{abstract}




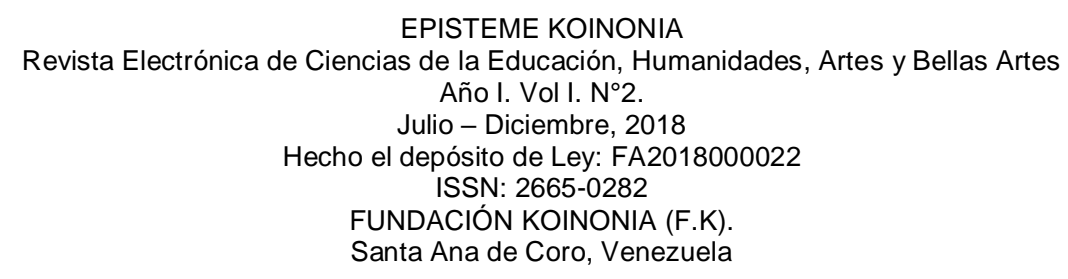

Raemy Roque Díaz

evidenced by the students of their emotions, in comparison with the initial moment of the investigation, however, it is pertinent to continue working with them in order to contribute to the achievement of an assertively emotional personality.

Descriptors: educational psychology, child psychology, educational guidance, counselling.

\section{INTRODUCCIÓN}

El diagnóstico permitió a la investigadora, estudiar la realidad en consonancia con el equipo de investigación que le acompaña, de ese modo, se realiza el análisis con la finalidad de trabajar de modo sistemático, la solución de los problemas detectados, teniendo en consideración que en la medida que se trabaja en equipo para la resolución de los mismos, se debe promover la transformación de los actores sociales que intervienen en el proceso investigativo, por consiguiente, Ander - Egg (2003) señala que "El principio fundamental en que se basa la necesidad de realizar un diagnóstico social es el de conocer para actuar" (p. 94). Por consiguiente, con el diagnóstico se obtiene la información concreta para realizar una intervención contribuyente a solventar no solo problemas, sino en planificar para trabajar en el crecimiento integral del ser humano.

Así mismo, en la investigación acción participativa, la información obtenida del diagnóstico, gestiona las respuestas a un problema o problemas en concreto. Ante lo cual, Ander - Egg (2003) señala que el diagnóstico se elabora con dos propósitos definidos:

En primer lugar, ofrecer una información básica que sirva para programar acciones concretas. Esta información será más o menos amplia, según se trate de elaborar un programa, proyecto, o simplemente realizar determinadas actividades. En segundo lugar, proporcionar un cuadro de situación que sirva para seleccionar las estrategias de actuación más adecuadas (p. 95). 


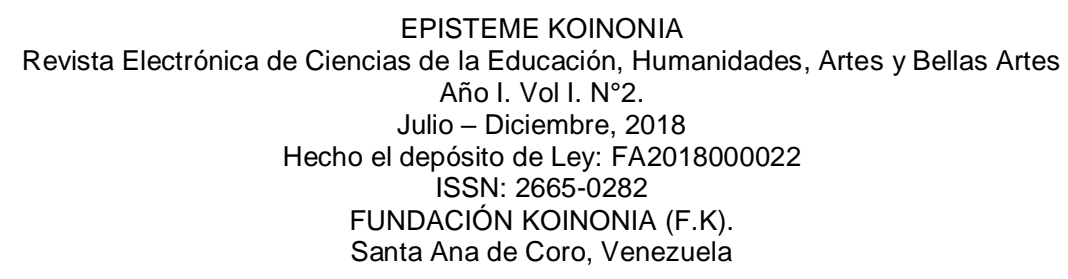

Raemy Roque Díaz

Partiendo de lo planteado, es impensable que la investigación acción participativa, pueda ser realizada efectivamente sí no se ha realizado un adecuado diagnóstico que proporcione la información necesaria para realizar una planificación que satisfaga la necesidad del grupo de investigación. Por consiguiente, para desarrollar un diagnóstico donde todos los estudiantes de sexto grado sección única, del turno de la tarde del CEDAIN - UNEFM participarán, se procedió a brindar el derecho de palabra (reunión de trabajo el día martes 11 de octubre de 2016), utilizando para ello la técnica de la lluvia de ideas, la cual permitió ir construyendo el diagnóstico de modo participativo, el diagnóstico participativo permite conocer necesidades con la finalidad de generar estrategias contextualizadas a la solución de las mismas, Reyes Aular (2016).

Otra estrategia utilizada, (reunión de trabajo el día martes 25 de octubre de 2016), fue la de mostrar un Papelógrafo con imágenes alusivas al desarrollo personal, entre las cuales se encontraban: autoestima, comunicación, liderazgo, generándose así un debate y reflexión sobre la importancia de formarse en estas dimensiones con el fin de crecer integralmente como persona, conociéndose a sí mismo y mejorar las relaciones con sus semejantes, a fin de propiciar un ambiente de aprendizaje cónsono en donde todos sus actores contribuyan al crecimiento personal, mediante un proceso sistémico proyectado desde la orientación educativa como eje transversal en el desarrollo de las actividades escolares y sociales de los estudiantes, siendo pertinente tener en cuenta en la formación de los docentes la preparación en abordaje emocional, Romero \& Villasmil (2017).

En aras de sistematizar las experiencias desarrolladas en el diagnóstico, se procedió a elaborar un árbol de problemas, (reunión de trabajo el día martes 22 de noviembre de 2016), con la finalidad de que todos los actores involucrados en el proceso de investigación (docente - estudiantes) pudiesen acordar y jerarquizar el problema a abordar durante el trabajo investigativo, de ese modo, todos tuvieron la oportunidad de participar de modo activo y ameno en la construcción de una visión general sobre las 


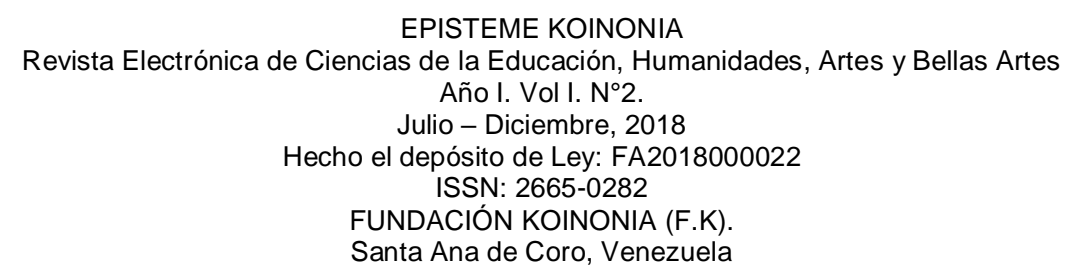

Raemy Roque Díaz

problemáticas en función de brindar apoyo al crecimiento personal y mejora de las relaciones interpersonales.

\section{Análisis del árbol de problemas}

Las problemáticas que afectan a los estudiantes del sexto grado del "Centro Experimental de Atención Integral del Niño CEDAIN - UNEFM", se refieren a las relaciones interpersonales por cuanto tienden a manejarse en ocasiones con agresividad verbal y física con sus compañeros, desmotivación por realizar las actividades académicas que se les encomiendan en clases, así como también se evidencia falta de visión para lograr metas, debido que no poseen un proyecto de vida que les oriente a la consecución de metas en el corto, mediano, largo plazo, así como también el escaso apoyo de las familias y representantes a superar estos problemas, permiten que las relaciones interpersonales entre los estudiantes en el ambiente de aprendizaje, no sean las más asertivas para crecer personal - colectivamente en beneficio de constituirse en una persona con visión integral de vida para transcender los problemas que puedan desarrollarse a lo largo de su vida.

Muchas pudiesen ser las causas que originan estos problemas, sin embargo, la raíz de los mismos se encuentra en la personalidad de cada uno de los estudiantes, por cuanto se evidencia una fuerte desmotivación hacia el logro, de ahí que se hace importante trabajar desde la persona, es decir, fomentar una mejor personalidad por cuanto esto puede ayudar a mejorar el rendimiento académico en todos los niveles, así como las relaciones interpersonales con sus semejantes, en la medida que se procura fomentar un liderazgo transformador en los estudiantes, de ese modo, desde la investigación bajo una visión de la orientación educativa se procura apoyar a un grupo de estudiantes a transcender como mejores personas, siendo pertinente involucrar a los padres y representantes en atención a los estudiantes, Hernández (2018).

Por lo tanto, hoy día se hace necesario en la educación trabajar todas las áreas en las cuales se desarrolla el ser humano (bio psico social espiritual) puesto que trabajando 


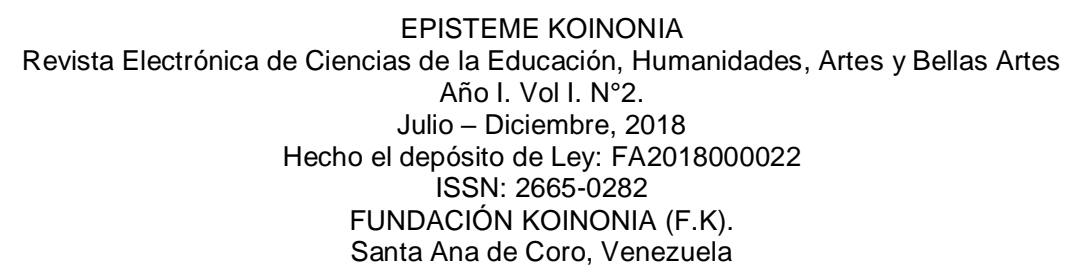

Raemy Roque Díaz

del mejor modo posible el desarrollo humano individual de los estudiantes se podrá contar con un colectivo que aporte lo mejor de sí al medio social donde se desenvuelve. De ahí, que el trabajar el fomento de una mejor personalidad se hace indispensable, puesto que la escuela no sólo se debe preocupar por un rendimiento académico, sino, que debe incentivar al logro de una mejor persona. El trabajar la personalidad de los estudiantes promueve la desaparición de la desmotivación que sufren en estos momentos y que desde luego sólo les conduce al fracaso tal como indican Mirete, Soro \& Maquilón (2015).

De esto se deriva que la desmotivación escolar no la produce un sólo factor, sino, que es una serie de factores los que ocasionan la misma, entre los cuales se pueden encontrar: problemas familiares, falta de un buen proceso de orientación, docentes que no inculcan valores, sino, sólo conocimientos en el aula de clases, los medios de comunicación social que hoy saturan el mercado con programas violentos y hasta censurable, son sólo alguna de las razones por las cuales se pudiese originar la desmotivación personal- escolar, se hace inherente atender esta necesidad a fin de contribuir en la formación de personas que hoy son niños y tienen mayor posibilidad de ser mejores personas en el futuro, sí se les apoya, asesora, orienta, adecuadamente para conocerse así mismo, por ende desarrollar relaciones interpersonales emocionalmente favorables para sí y sus semejantes. Idea inicial de investigación Partiendo de los problemas descritos anteriormente, la presente investigación procura realizar un plan de acción donde se trabaje la personalidad de los estudiantes, mediante el fomento de las relaciones interpersonales, por cuanto de este modo se estará atacando la problemática desde su raíz, debido que el plan contempla trabajar el auto conocimiento de los estudiantes mediante la transmisión de valores afectivos, sociales, espirituales, que le permitan convertirse en mejores personas.

De lo contrario se corre el riesgo que estos estudiantes puedan profundizarse en caminos que no son los más convenientes para el desarrollo integral de la persona, sino, que lo conducen a su destrucción. Por lo tanto, la situación en que se encuentran 


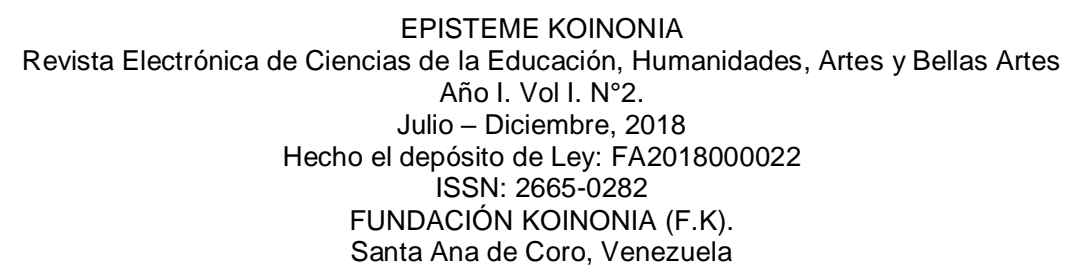

Raemy Roque Díaz

los estudiantes del sexto grado del "Centro Experimental de Atención Integral del Niño CEDAIN-UNEFM", es preocupante debido que el bajo rendimiento académico no es el problema en sí que padecen, sino, la desmotivación que proviene de una baja identidad como personas y que se desencadena en relaciones interpersonales no cónsonas para el aprendizaje cooperativo en aras de crecer integralmente como persona para en el futuro ser un ciudadano al servicio de la sociedad venezolana.

Se puede decir que los estudiantes se encuentran sentados bajo una bomba de tiempo que pueda estallar en cualquier momento por cuanto si en el hogar su estima no es tratada del mejor modo, en el CEDAIN - UNEFM tampoco se hace lo pertinente para lograr una mejor personalidad, esto hace que los estudiantes se conviertan en una presa fácil de desviarse del buen camino por cuanto no tendrán valores claros que le permitan armarse contra los ataques que la sociedad postmoderna promueve hoy en día, tales como sexo desenfrenado, drogas, consumismo, irrespeto a la autoridad de cualquier tipo, deserción escolar, baja calidad en las actividades escolares, entre otros, que pueden afectar el buen desarrollo integral de los estudiantes.

Se hace imperioso abordar esta problemática (relaciones interpersonales) que se detecta en el "Centro Experimental de Atención Integral del Niño CEDAIN-UNEFM, específicamente en el sexto grado (turno de la tarde) por cuanto no sólo se trata de que los estudiantes puedan motivarse para incrementar su rendimiento académico o para ser mejores estudiantes, sino, que deben convertirse en mejores personas para lograr el éxito en sus vidas por medio de los valores éticos y morales que engrandecen al ser humano.

Sólo de este modo se estará contribuyendo en solucionar una problemática que afecta a un grupo de estudiantes pero que a su vez se convierte en el reflejo de la sociedad. Los docentes no pueden estar a espalda de esta realidad, sino, que por el contrario deben abocarse a activar mecanismos que permitan atacar la problemática presentada en la institución, de ahí que en conjunto (investigadora - institución) se haya acordado 


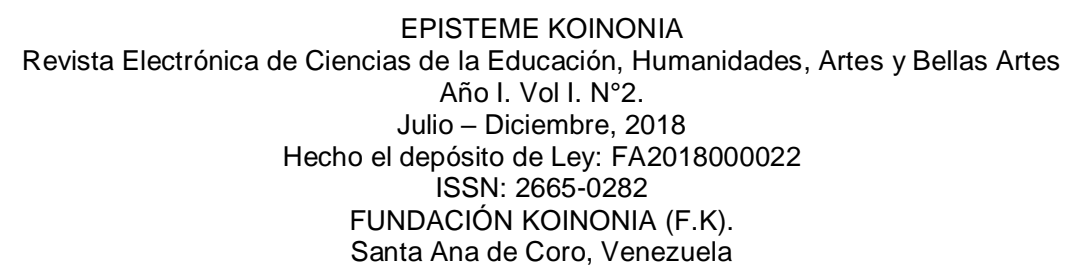

Raemy Roque Díaz

trabajar en un plan que incremente la personalidad de los estudiantes y así se minimice la problemática de desmotivación que padecen los estudiantes.

Esta falta de desmotivación se traduce en escaza o nula participación en el aula de clases, poca preparación de los temas que son abordados en las clases, es decir, que los estudiantes no se preocupan por preparar las actividades que los docentes les encomiendan del mejor modo posible, otro aspecto que se evidencia es la baja estima que padecen algunos estudiantes lo que ocasiona timidez en los mismos, lo que les impide tener una mejor participación en el aula de clases, el estudio literal de los contenidos expuestos en clases es otra de las problemáticas encontradas aunado a la conducta de los estudiantes que muchas veces no es la mejor.

Mejorar las relaciones interpersonales en su conjunto en los estudiantes de sexto grado del Centro Experimental de Atención Integral del Niño CEDAIN-UNEFM, es la visión inicial de la presente investigación, por cuanto no se trata de apoyar en la transformación de un problema exclusivo alusivo a la personalidad, sino, desarrollar en su conjunto para que estas puedan ser mejoradas y por ende se consolide una personalidad acorde las exigencias que la sociedad venezolana demanda hoy día.

Es decir, es contribuir en formar al estudiante desde su crecimiento personal, hacia la toma de conciencia sobre la importancia que juega en ser una persona que se auto conozca, maneje sus emociones favorablemente, pueda relacionarse excelentemente con sus semejantes, debido que esto es la base para triunfar en el mundo competitivo de hoy, por consiguiente desarrollar asertivas relaciones interpersonales a partir del propio manejo efectivo de sí mismo, constituye un pilar fundamental para convertirse en una persona de éxito de modo integral. En este sentido, la Organización para la Cooperación y el Desarrollo Económico (OECD) (2016) plantea que

Al graduarse, los niños y los jóvenes en las escuelas de todo el mundo se enfrentarán a un futuro muy diferente del de las generaciones anteriores. Los avances tecnológicos y los descubrimientos científicos están acelerando de manera significativa la cantidad de conocimiento e información disponible. Ahora vivimos en una comunidad internacional cada vez más 


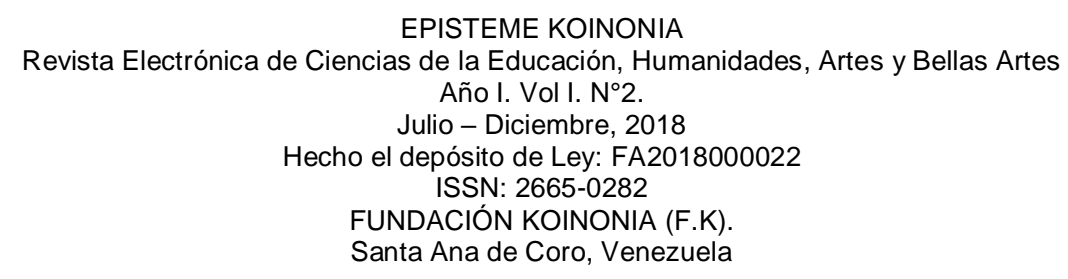

Raemy Roque Díaz

interdependiente, en la que el éxito o el fracaso de un país tiene consecuencias para muchos otros (p. 15).

Desde lo planteado, el rol de la escuela tradicional donde solo se imparten conocimientos, debe transcender hacia una donde se anexe el trabajo emocional de los estudiantes, esto contribuirá a mejorar las relaciones interpersonales en aras de contar con un ambiente de aprendizaje armónico, el cual debe ser reflejo de la sociedad donde se desenvolverá el estudiante como ciudadano. Esto forma parte una educación con base en la orientación educativa como perspectiva para promover no solo un mejor aprendizaje, sino el crecimiento integral de la persona.

En este sentido, el papel del maestro debe ser holístico en función de promover no solo un excelente aprendizaje, pensamiento crítico, sino, la formación de valores en el estudiante que le permitan socializar asertivamente consigo mismo y sus semejantes, esto permite que la escuela se convierta en un lugar donde el estudiante, obtiene la orientación necesaria para convertirse en una persona de bien, de excelencia, pero sobre todo, donde se alfabetice emocionalmente.

La investigación tuvo como propósito principal: Desarrollar un plan para el desarrollo personal y manejo asertivo de emociones en estudiantes del CEDAIN UNEFM.

\section{METODOLOGÍA DE LA INVESTIGACIÓN}

La presente investigación siguió los lineamientos de la teoría crítica o paradigma sociocrítico, un modelo epistemológico que, entre otras cosas, plantea el abordaje de una realidad compartida, histórica, construida, dinámica, múltiple, holística, divergente, teniendo como principal interés emancipar a los sujetos participantes, identificando para ello el potencial para el cambio a través del análisis de dicha realidad. Específicamente, este enfoque se trabajó desde lo propuesto por Martínez (2006).

En este orden de ideas, la investigadora cuando trabaja bajo el enfoque socio crítico genera situaciones de encuentro con las personas involucradas en la investigación con el propósito de que reflexionen sobre la situación que acontece y así entre todos los 


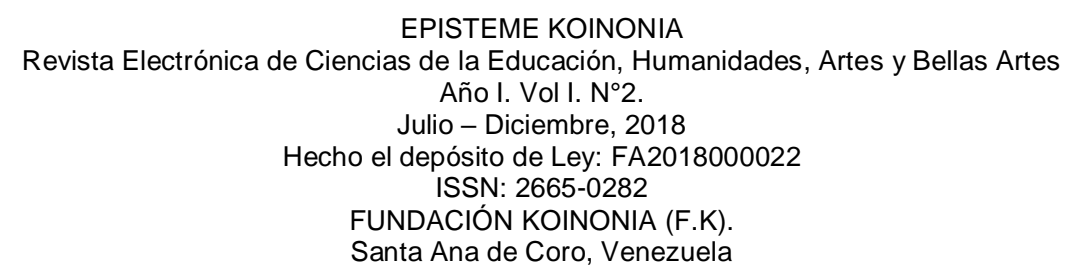

Raemy Roque Díaz

involucrados como un equipo trabajar en busca de la solución del problema. Asimismo, de acuerdo con la citada fuente, para alcanzar tales propósitos se vale de la aplicación de estudios de casos y técnicas dialécticas por parte de un investigador fuertemente comprometido con el cambio y dispuesto a convertirse en parte del problema.

En líneas generales la metodología que propone este paradigma es dialógica e interactiva, buscando eliminar la falsa conciencia, promover y facilitar la transformación. Siguiendo tales criterios, esta teoría investigativa resulta particularmente útil para la investigación acción participante (IAP), la cual entre sus rasgos contempla la indagación sistemática y mantenida, planificada y autocrítica, la cual está orientada por la práctica socio pedagógica porque surge de ella y a ella se devuelve, esto con el propósito de comprenderla para lograr cambiar la realidad y reconstruirla.

Por otra parte, de acuerdo a las características del paradigma de la teoría crítica o paradigma socio crítico y los rasgos señalados de la investigación acción participante, se considera que el presente estudio es del tipo investigación acción, denominada por Martínez (2006), como "el conjunto de principios, normas y procedimientos metodológicos que permiten obtener conocimientos colectivos sobre una determinada realidad social" (p.34) y tomando en cuenta tal conjunto se le proporcionará herramientas con el fin de promover la formación de conflictos comunitarios.

\section{Fases de la investigación}

La investigación acción participativa de acuerdo a Bisquerra (2004, p. 34) cumple con las fases de: "diagnostico, diseño de la propuesta, aplicación y evaluación de la propuesta de intervención", de ahí que la presente investigación posea las siguientes fases:

Diagnóstico: Permite realizar la inserción a la comunidad de estudio, conocer sus potencialidades, debilidades, problemáticas, de viva voz de los actores sociales, de ese modo, puede desarrollarse una relación entre el investigador y las personas que participan en la investigación, los cuales se convierten en co investigadores. 


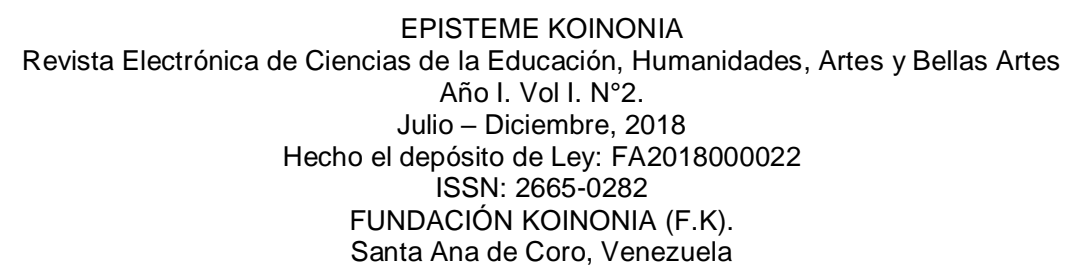

Raemy Roque Díaz

Diseño de la propuesta: Una vez analizado los problemas en conjunto con el equipo de investigación se procede a realizar una propuesta de trabajo basada en formación para la resolución de conflictos comunitarios.

Aplicación de la propuesta: Se aplicará una propuesta de trabajo basada en formación para la resolución de conflictos comunitarios.

Evaluación de la propuesta: Esta fase permite a los investigadores evaluar el impacto que ha dejado la investigación en la comunidad. La evaluación se realizará siguiendo la sugerencia metodológica de Ander - Egg (2003, p. 111) quién expresa que para la evaluación del proyecto ejecutado, los investigadores respondan las siguientes interrogantes:

¿Qué hicimos? Se refiere al grado y forma en que se lograron los objetivos propuestos.

¿Qué aprendimos con el proyecto realizado? Se refiere a la experiencia o conocimiento alcanzado por los investigadores por medio de la interacción con las personas que participaron en el proyecto, por cuanto existió un intercambio de saberes y experiencias que enriquecen el crecimiento personal y profesional.

\section{Descripción del grupo abordado en la investigación}

El grupo abordado en la investigación se encuentra integrado por 15 niños en edades comprendidas entre los ocho y nueve años de edad, ocho (08) varones y siete (07) hembras que estudian el sexto grado de educación primaria en el CEDAIN - UNEFM, turno de la tarde. Estos niños presentan características físicas, emocionales, acordes a la edad en que se encuentran, sin embargo, presentan rasgos de timidez cuando la docente les interroga sobre aspectos relacionados al estudio o actividades académicas, de desmotivación hacia los estudios, de agresividad hacia sus compañeros a la hora de establecer relaciones interpersonales, lo que desde luego les afecta en su rendimiento académico y en el modo de manejar sus emociones consigo mismo y demás estudiantes. 


\section{RESULTADOS}

\section{Fases de sistematización de la información}

En este sentido, la investigadora observó y sistematizó los aspectos cognitivos, conductuales, de los estudiantes, durante el desarrollo de las sesiones o encuentros, generándose del siguiente modo:

El 25 de octubre 2017 se llevó a cabo el primer encuentro. Luego de explicar a los estudiantes el motivo del trabajo investigativo, mostrarles el plan de actividades correspondientes a las diez sesiones; se dio inicio a la clase con una dinámica rompe hielo denominada "Aun si te equivocas" para crear un clima ameno en el aula.

Posteriormente se comenzó a estudiar la teoría relacionada con la autoestima, inteligencia emocional y motivación para lo cual en primera instancia se realizó un torbellino de ideas de manera que los estudiantes expresaran su punto de vista, sin embargo no se logró la participación de todo el grupo, pues algunos mostraron una actitud apática en la actividad, no obstante las pocas ideas aportadas permitieron ahondar en la temática.

Seguidamente se explicaron las teorías ejemplificando cada uno de los temas, con lo que se fue incentivando la participación de los estudiantes, quienes luego discutían en

grupo el contenido de la clase y algunas expresiones con sus propias palabras la temática abordada.

\section{Resumen}

Actitud apática ante las actividades.

No obstante a las estrategias empleadas se evidencia muy poca participación de los estudiantes.

Poco dominio del contenido teórico. 


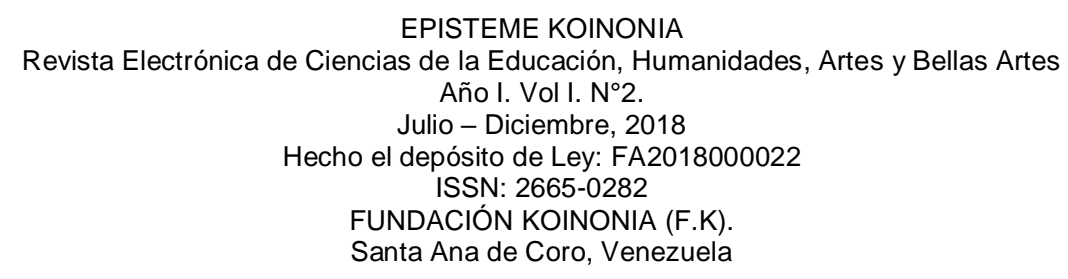

Raemy Roque Díaz

El día 26 de octubre de 2017 se llevó a cabo la segunda sesión de clase en la que se estudiaron las siguientes teorías: liderazgo, comunicación y valores. Para iniciar se utilizó la técnica de resumen por medio de la cual los estudiantes expusieron los conocimientos adquiridos en la clase anterior, para este día hubo mayor participación por parte de los estudiantes, seguidamente explicamos detalladamente cada una de las teorías correspondientes y se les hizo entrega de un material en el que se evidenciaban los objetivos del liderazgo, la importancia de la comunicación y lo imprescindible que es educar y educarse en valores.

Resumen

Hubo un poco más de participación por parte de los estudiantes.

Se logró que los estudiantes adquirieran confianza de expresar sus inquietudes e ideas.

El día 27 de octubre de 2017 se llevó a cabo el tercer encuentro con los estudiantes, comenzamos a leer y analizar la obra "El caballero de la armadura oxidada", desde el inicio de la clase los estudiantes se mostraron interesados en conocer el contenido de la novela. En un principio participaron en una estrategia denominada "conéctate con tus sentimientos", por medio de la cual evidenciaron la importancia de demostrar y recibir afecto, luego procedieron a leer la novela y a establecer relación entre el texto y las teorías estudiadas anteriormente.

Con estas actividades se logró que los alumnos se esforzaran para el cumplimiento de los mismos y captaron con mayor precisión el contenido implícito en el texto, además manifestaron interés por conocer el desarrollo y desenlace de la historia.

Resumen

Los estudiantes se mostraron motivados al realizar las actividades.

Comprendieron el contenido de la clase de manera efectiva.

Mostraron interés por adquirir mayor conocimiento. 


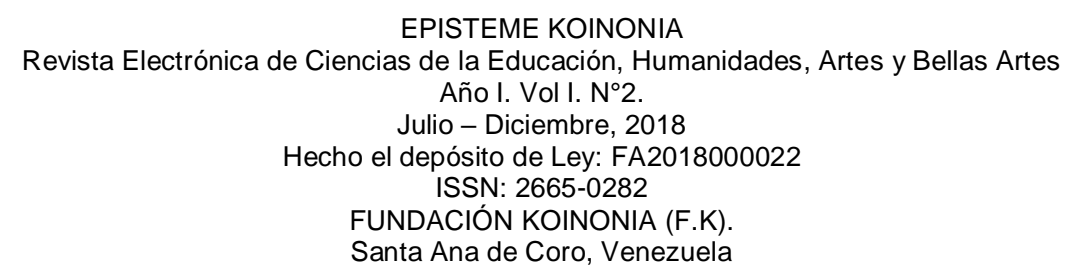

Raemy Roque Díaz

El día 01 de noviembre de 2017 se llevó a cabo la cuarta sesión de clase, en ella estudiamos el segundo capítulo de la obra denominada "En los bosques de Merlín". Con la intención de motivar a los estudiantes a participar en la clase, se aplicó la técnica de repaso de manera que los estudiantes proyectaran su atención en la actividad.

Se procedió a leer el capítulo correspondiente, luego los alumnos realizaron en grupos; mapas mentales en los que pusieron de manifiesto su creatividad reflejando el contenido del texto a través de ilustraciones, seguidamente expusieron a sus compañeros el trabajo realizado. Para este momento había más confianza entre todos y se respetaba un óptimo ambiente en el aula. Se logró el objetivo propuesto de manera satisfactoria.

Resumen

Los estudiantes estuvieron atentos a la clase.

Trabajaron en equipo y manifestaron su creatividad.

Expresaban sus ideas sin temor a equivocarse.

El día 02 de noviembre de 2017 se llevó a cabo la quinta sesión de clase en la cual se dio lectura al tercer capítulo de la obra denominada "El sendero de la humanidad", en este día los estudiantes presentaron una actitud crítica desde el inicio, cada uno expresó su punto de vista de acuerdo al tema tratado, participaron en las actividades, pues realizaron un mapa conceptual en el que plasmaron las ideas que consideraron relevantes y luego lo expresaron al resto de sus compañeros por lo que se pudo evidenciar que hubo comprensión de la lectura.

Resumen

Demostraron una actitud crítica.

Todos participaron en las actividades realizadas.

Comprendieron el texto y expresaron sus ideas. 


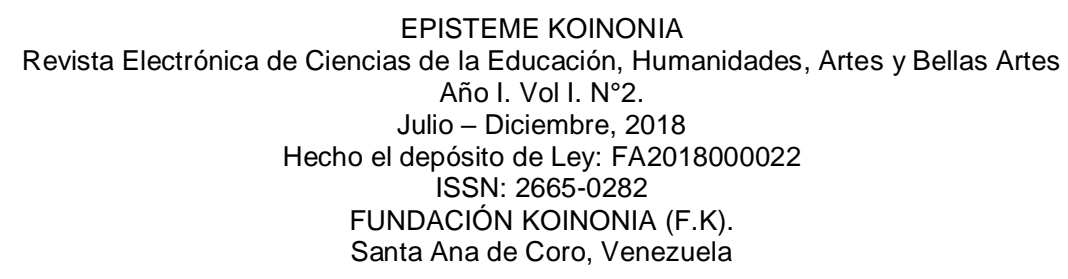

Raemy Roque Díaz

El día 03 de noviembre de 2017 se realizó la sexta sesión de clase, en la cual se leyó y discutió el cuarto capítulo de la obra del Caballero de la Armadura Oxidada denominada "El Castillo del Silencio", en esta sesión los alumnos se mostraban cada vez más entusiasmados por la lectura y motivados a participar. Muchos de ellos contaron anécdotas personales relacionándolos de una forma $u$ otra con el capítulo leído estableciendo semejanza entre ambas, se desarrolló la clase de manera que al realizar el análisis plasmaron con mayor facilidad sus ideas por cuanto asimilaron el contenido notablemente.

Resumen

Los estudiantes se mostraron motivados e interesados en aprender y participar en las actividades.

Desarrollaron su habilidad al establecer semejanzas entre el texto y sus experiencias.

El día 08 de noviembre de 2017 se discutió el quinto capítulo de la obra denominada "El castillo del conocimiento", primeramente se hizo un repaso de la clase anterior y luego se comenzó a leer y analizar el texto detalladamente en esta sesión los estudiantes seguían mostrándose motivados, participativos e integrados por conocer lo que acontecería al final de la historia, en este día comprendieron la importancia que amerita poseer conocimientos y crecer interiormente cada día a día.

Resumen

Los estudiantes manifestaron su interés y motivación.

Participaron en las actividades realizadas.

Comprendieron lo importante de su desarrollo personal.

El día 09 de noviembre de 2017 se lleva a cabo la octava sesión de clase en la que se leyó el VI capítulo de la novela denominada "El castillo de la voluntad y la osadía" en primera instancia se aplicó una estrategia llamada "Que recuerdos de mi" para 


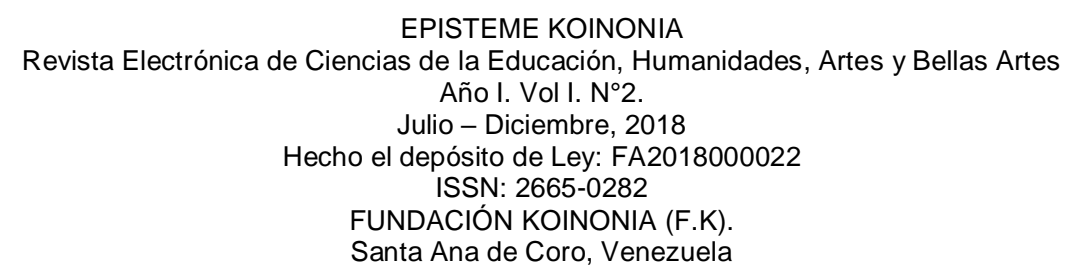

Raemy Roque Díaz

retomarlas ideas expuestas en su clase anterior y lograr de esta manera la participación del grupo.

Luego se procedió a leer y analizar el contenido del capítulo correspondiente para que posteriormente los estudiantes realizaran una producción escrita, actividad en la que expusieron el aprendizaje adquirido, en este día hubo mucha participación, se evidenció que captaron el mensaje implícito en el texto, además expusieron situaciones en las que han tenido dudas y sentir miedo, así como también dieron a conocer su punto de vista con respecto a la importancia de demostrarse a sí mismo hasta donde somos capaces de llegar.

Resumen

Todo el grupo participó, llevaron a cabo las actividades de manera satisfactoria.

Expresaron sus dudas y miedos.

Comprendieron lo importante que amerita el poseer voluntad y osadía para el logro de nuestras metas.

El día 10 de noviembre de 2017 se realizó la novena sesión de clases en la que se leyó y analizó el séptimo y último capítulo de la obra "El caballero de la Armadura Oxidada"; primeramente retomamos de manera resumida el contenido de la obra para este entonces, los estudiantes interesados en conocer el final de la historia predecían algunos posibles desenlaces, seguidamente procedieron a leer el capítulo y por medio de la estrategia comprendieron y realizaron un análisis en el que plasmaron además del contenido lo relevante y satisfactorio que es proponerse metas y luchar por alcanzarlas venciendo los obstáculos que se interponen.

Resumen

Los estudiantes demostraron una actitud dinámica y activa.

Pusieron en práctica su capacidad de análisis. 


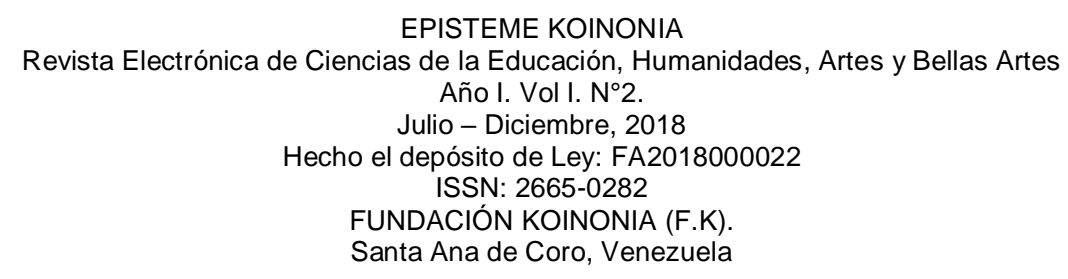

Raemy Roque Díaz

El día 16 de noviembre de 2017 se llevó a cabo la décima sesión de clase en la cual los estudiantes presentaron una lectura dramatizada de la obra "El Caballero de la Armadura Oxidada" primeramente preparada y ensayada. En esta actividad los alumnos pusieron en práctica su creatividad, con entusiasmo y motivación alcanzaron un óptimo desempeño con lo que mostraron el aprendizaje adquirido y a través del cual se pudo evidenciar el logro de los objetivos propuestos.

Resumen

Dieron a conocer su habilidad en cuanto a originalidad y creatividad.

Participaron en las actividades eficientemente.

\section{ANÁLISIS CUALITATIVO}

Los resultados obtenidos de la aplicación de un plan de acción para fomentar el desarrollo personal y manejo asertivo de las emociones en estudiantes del sexto grado del CEDAIN - UNEFM, demostraron que en la medida que se toman en cuenta las necesidades e intereses del educando y se implementan actividades motivacionales que le despierten el interés por adquirir conocimientos, el estudiante logra crecer como ser humano integral acorde a las exigencias de la sociedad.

Al llevar a cabo el plan de acción propuesto se logró que los estudiantes participaran en las actividades y dejaran de lado la actitud apática que venían mostrando, expresaron sus ideas y aclararan sus dudas, llevaron a cabo las actividades asignadas cumpliéndolas con eficiencia y dieron a conocer sus necesidades de recibir valores afectivos así como también su creatividad.

Los cambios logrados en la actitud de los estudiantes fueron evidenciados por los docentes de la institución quienes dieron a conocer su satisfacción por el logro de los objetivos y comprendieron lo importancia de brindar conocimientos través de actividades placenteras que incentiven al estudiante en cada actividad. 


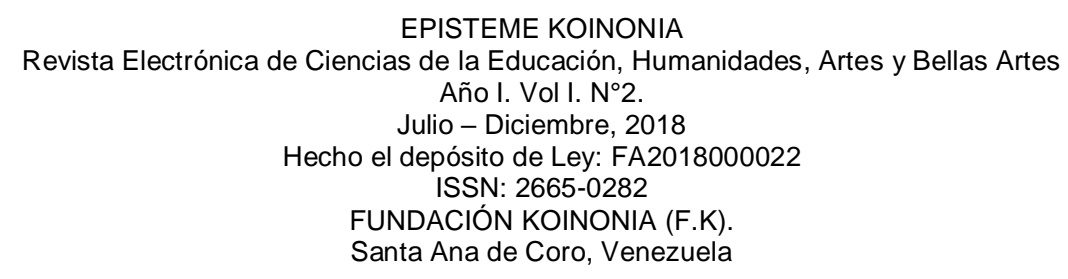

Raemy Roque Díaz

\section{Proceso de triangulación}

En función de generar la validez de la investigación, se construyó una triangulación de datos Ander - Egg (2003, p. 18), mediante la cual, se contrastaron datos teóricos, datos provenientes de las sesiones realizadas y observación por parte de la investigadora, en este sentido se presenta:

\section{Datos teóricos}

El desarrollo personal o crecimiento personal de acuerdo a Escobar (2012, p. 16) se entiende por "la actualización de las potencialidades humanas (psicológicas y espirituales) que la persona puede hacer más allá de su desarrollo natural en función de la edad". Por lo tanto, esto trae como beneficio que persona la persona aprende, a través de la conciencia de sí mismo, a aprovechar sus posibilidades de pensar, sentir y actuar para: Usar el pensamiento libre o autónomo, dominar una libertad responsable, siendo líder de sí mismo y tener salud emocional.

Por consiguiente, desde la orientación educativa se hace necesario formar para que los estudiantes conozcan y manejen sus emociones, siendo así una oportunidad para mejorar las relaciones consigo mismo y sus semejantes, de ese modo, se podrá contar con ambientes de aprendizaje con educación emocional acorde al nivel de estudio y etapa en la cual se encuentran los estudiantes, esto permitirá fomentar mejores relaciones, así como un aprendizaje cooperativo donde se construyan los significados de los temas que se abordan, siendo así más significativa la educación.

\section{Datos de las sesiones}

Los estudiantes demostraron una actitud dinámica y activa.

Pusieron en práctica su capacidad de análisis. 


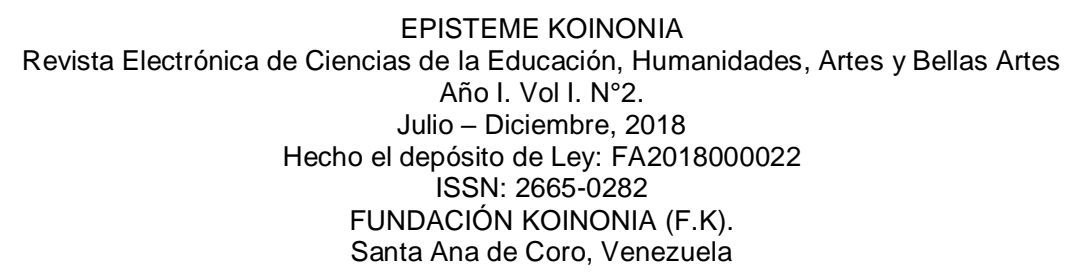

Raemy Roque Díaz

Dieron a conocer su habilidad en cuanto a originalidad y creatividad.

Participaron en las actividades eficientemente.

\section{Datos de la investigadora}

Se basó en la observación realizada a lo largo de la investigación, en donde al inicio se evidenciaba que existía apatía por parte de estudiantes para desarrollar activamente sus actividades escolares, así como relacionarse con otros compañeros de modo asertivo. De ese modo, se trabajó en un proceso de diez sesiones o encuentros, en donde por medio del estudio teórico sobre desarrollo personal y emociones como referenciales teóricos, se procedió a la lectura, reflexión, de la obra el caballero de la armadura oxidada, fábula que permitió a los estudiantes motivarse a leer y comprender lo importante contar con un desarrollo personal, de las emociones, de modo asertivo, dado que esto le permite al ser humano ser mejor persona cada día, en la medida que se auto conocen y aceptan.

\section{Evaluación a los participantes}

Los participantes, estudiantes del sexto grado del colegio CEDAIN - UNEFM, en relación al momento inicial en el cual se encontraban en la investigación, lograron en líneas generales crecer personalmente, dado que hoy no solo tienen un manejo cognitivo del tema sobre desarrollo personal y manejo asertivo de las emociones, sino que comprenden la importancia que tiene el leer libros relacionados al crecimiento personal de modo constante con la finalidad de poder formar una personalidad asertiva al manejo de los conflictos personales, sociales, a los cuales puedan enfrentarse.

De ese modo, puede señalarse como beneficioso este primer aporte investigativo a los estudiantes, dejando la posibilidad de seguir trabajando con ellos en potenciar aún más sus habilidades emocionales, dado que no puede darse por hecho de que ya se encuentran en un nivel que les permita auto conocerse totalmente, más aun cuando 


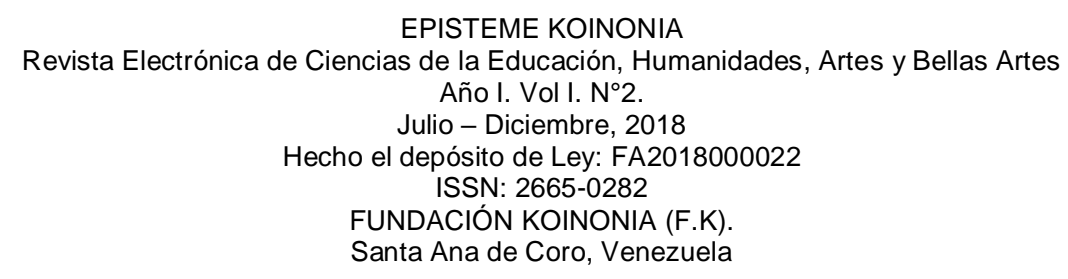

Raemy Roque Díaz

próximamente se enfrentarán a cambios como la adolescencia y sistema educativo, situaciones que inciden en la personalidad.

En ese sentido, es pertinente seguir trabajando con los estudiantes en el desarrollo personal, para lo cual es necesario proseguir con la investigación acción, y no darla del todo concluida, aunque se presenta un primer avance de la misma que servirá para cumplir con requisitos académico de grado, en la realidad escolar del día a día, a pesar de lo beneficiosa que ha sido la investigación, es pertinente seguir trabajando con los estudiantes a lo largo del año escolar, con la finalidad de prepararlos cognitivamente a los nuevos desafíos que han de enfrentar como personas que se encuentran en evolución biológica y física.

\section{CONCLUSIONES}

\section{Reflexionar con los estudiantes sobre las necesidades y problemas de desarrollo personal y manejo asertivo de emociones.}

Se reflexionó con los estudiantes en función de contar con un diagnóstico inicial de las necesidades y manejo asertivo de emociones, para lo cual se partió de las siguientes problemáticas:

No tienen claridad de metas a lograr en la vida. No poseen proyecto de vida

Agresividad con los compañeros

Baja autoestima que no permite manejar adecuadamente el bullying

De ese modo, se direccionó un proceso en donde se realizaron conversatorios sensibilizadores sobre la importancia de superar tales problemáticas, para lo cual se incentivó a los estudiantes que participaran en una investigación en donde se iban a abordar estrategias que contribuyeran en transcender las problemáticas planteadas, en la medida que se generaba el desarrollo personal y manejo asertivo de las emociones, esto les motivó a participar dado que era una oportunidad para crecer integralmente como personas, generándose la reflexión sobre lo importante que es conocerse así mismo para ser una mejor persona. 
EPISTEME KOINONIA

Revista Electrónica de Ciencias de la Educación, Humanidades, Artes y Bellas Artes

Año I. Vol I. №2.

Julio - Diciembre, 2018

Hecho el depósito de Ley: FA2018000022

ISSN: 2665-0282

FUNDACIÓN KOINONIA (F.K).

Santa Ana de Coro, Venezuela

Raemy Roque Díaz

\section{Planificar estrategias que alcancen el manejo asertivo de las emociones en el desarrollo personal de los estudiantes.}

Se planificaron estrategias de desarrollo personal y manejo asertivo de las emociones desde la lectura del libro el caballero de la armadura oxidada, por cuanto esta es una fábula que aborda el crecimiento personal desde la fantasía, constituyéndose en una lectura motivadora para los estudiantes, por consiguiente este libro permite al lector crecer en la medida que se identifica con los personajes y las vivencias que estos desarrollan en la trama, generándose una oportunidad de cambio favorable.

\section{Ejecutar estrategias que alcancen el manejo asertivo de las emociones en el desarrollo personal de los estudiantes.}

Las estrategias se ejecutaron mediante diez sesiones, siendo las dos primeras abordadas desde referentes teóricos, y el restante desde la lectura del libro el caballero de la armadura oxidada, de ese modo, en cada encuentro se relacionaba la lectura con el referencial teórico, mediante un proceso reflexivo de articular teoría con la práctica social de los estudiantes. Así mismo la investigadora generaba en cada encuentro estrategias estimuladoras de la partición individual, grupal, para que existiese una participación protagónica en cada encuentro y cada estudiante pudiese reflexionar sobre la importancia del desarrollo personal, así como del manejo asertivo de las emociones.

Evaluar las estrategias aplicadas para el manejo asertivo de las emociones en el desarrollo personal de los estudiantes. 


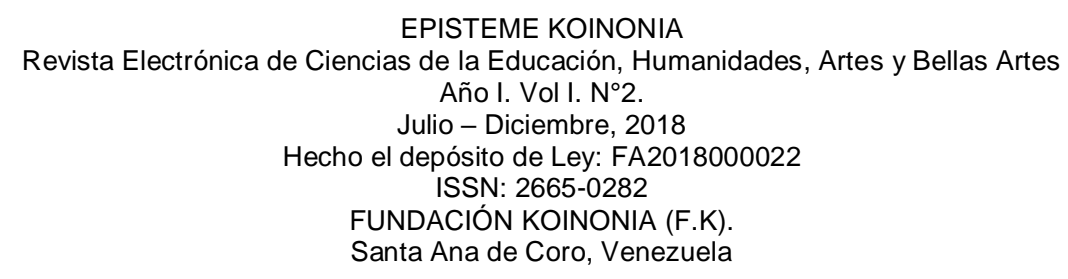

Raemy Roque Díaz

Al cierre de las sesiones, se realizaron conversatorios y observaciones con los estudiantes, evidenciándose lo siguiente:

Los estudiantes demostraron una actitud dinámica y activa.

Pusieron en práctica su capacidad de análisis.

Dieron a conocer su habilidad en cuanto a originalidad y creatividad.

Participaron en las actividades eficientemente.

Esto evidencia un crecimiento entre el momento inicial y de cierre de la investigación, sin embargo es pertinente seguir trabajando con los estudiantes en el fomento de su crecimiento personal, dado que pronto egresarán al primer año de educación básica, así como entraran en la edad adolescente, lo cual representa cambios a los cuales deben adaptarse en el mejor de los sentidos, para contribuir en superar los traumas propios de esta etapa de un modo favorable.

\section{REFERENCIAS}

1. Ander - Egg, E. (2003). Repensando la investigación acción participativa. Grupo Editorial Lumen. Buenos Aires, Argentina.

2. Bisquerra, R. (2005). Metodología de la investigación educativa. Editorial La Muralla. S.A. Barcelona, España.

3. Hernández, A. (2018). Transitando por el camino de la escuela para padres, madres y representantes. Una experiencia vivida. EPISTEME KOINONIA, 1(1), 51-71. Recuperado de https://fundacionkoinonia.com.ve/ojs/index.php/epistemekoinonia/article/view/490

4. Martínez, M (2006). Ciencia y arte de la metodología cualitativa. 2da edición. México: Trillas.

5. Mirete, A.B., Soro, M. \& Maquilón, J.J. (2015).El fracaso escolar y los enfoques de aprendizaje: medidas para la inclusión educativa. Revista Electrónica Interuniversitaria de Formación del Profesorado, 18(3), 183--196.DOI: http://dx.doi.org/10.6018/reifop.18.3.239021

6. OECD (2016). Mejorar el liderazgo escolar. Recuperado a partir de 
EPISTEME KOINONIA
Revista Electrónica de Ciencias de la Educación, Humanidades, Artes y Bellas Artes Año I. Vol I. N².
Julio - Diciembre, 2018
Hecho el depósito de Ley: FA2018000022
ISSN: 2665-0282
FUNDACIÓN KOINONIA (F.K)
Santa Ana de Coro, Venezuela

Raemy Roque Díaz

https://www.oecd.org/edu/school/43913363.pdf.

7. Reyes Aular, M. (2016). Programa de estrategias pedagógicas dirigido a los docentes para la participación de la familia al proceso de aprendizaje en la atención educativa no convencional. Revista Arbitrada Interdisciplinaria Koinonía, 1(2), 50-73. Recuperado de https://fundacionkoinonia.com.ve/ojs/index.php/revistakoinonia/article/view/39/27

8. Romero, M., \& Villasmil, J. (2017). Repensar la formación docente. hacia el encuentro de una nueva perspectiva epistémica para su abordaje y resignificación. CIENCIAMATRIA, $3(5), \quad 133-149$. https://doi.org/10.35381/cm.v3i5.17

C 2018 por los autores. Este artículo es de acceso abierto y distribuido según los términos y condiciones de la licencia Creative Commons Atribución-NoComercial-Compartirlgual 4.0 Internacional (CC BY-NC-SA 4.0) (https://creativecommons.org/licenses/by-nc-sa/4.0/). 